migrating into the duodenal wall. We concluded this was not related to the technique of PDS and should not deter us from continuing this practice. As expected our complication rates are higher that our units normal complication rates which reflects the challenging nature of the cases when PDS is attempted. Disclosure of Interest None Declared.

\section{PTH-011 CHANGING TRENDS OF ERCP, A DECADE APART}

K Hameed*. Gastroenterology, Good Hope Hospital Rectory Road, Sutton Coldfield Birmingham B75 7RR, Birmingham, UK

\subsection{6/gutjnl-2014-307263.457}

Introduction ERCP has become a well established therapeutic intervention. Indications and practice of ERCP have changed over the years due to advent of new imaging modalities. Our aim was to evaluate the ERCP indications, diagnosis and complications over 12 month period, a decade apart.

Methods Retrospective observational audit, data extracted from endoscopy reporting system and electronic patient's records. Consecutive patients undergoing ERCP over 12 months in 2002 and 2012.

Results Total number of ERCP's performed was 233 and 212 in the year 2012 and 2002. Median age was 73 and 69 with IQR $(58-82)$ and (56-80) years respectively. Gender ratio in 2012 of 1:1.1 and $1: 1.5$ in 2002 .

Diagnosis: in year 2012: stone disease 109 (46\%). Pancreatic Cancer 31 (13.7\%). Normal biliary tree 22(9\%)whilst in the year In the year 2002 stone disease 90(42\%). Pancreatic Cancer 16(7.5\%). Normal Biliarytree 60(28\%).

Interventions: Year 2012: stent insertion 72(30.9\%) and sphincterotomy 155(66.5\%). year 2002 stent insertion 39 (18.3\%) and sphincterotomies 86(40.6\%).

Technical success rate in both the years was $95 \%$.

Complications: 30 day mortality in 2012 was 22(9.4\%)and $12(5.7 \%)$ in 2002 . There was one procedure related death in year $2012(0.4 \%)$, none in 2002. Bleeding requiring transfusion : $14(6 \%)$ in $20126(2.6 \%)$ in 2002. Pancreatitis 6(2.6\%) and 6 $(3.3 \%)$ in 2012 and 2002. Seven patients required sedation reversal in year 2002 as opposed to 0 in 2012. Reflecting the significantly reduced doses being used in the latter year.

Conclusion 1) Work load remained the same. 2) Less number of normal ERCP'S 3) More pancreatic malignancies identified 4) More therapeutic procedures undertaken.

Disclosure of Interest None Declared.

\section{PTH-012 RESPONSE TO PERI-AMPULLARY BOTOX INJECTION AND SUBSEQUENT LONG TERM RESPONSE TO ENDOSCOPIC SPHINCTEROTOMY IN TYPE 2/3 SPHINCTER OF ODDI DYSFUNCTION}

K Wheeler*, CA Salmon, AS Austin. Gastroenterology and Hepatology, Royal Derby Hospital, Derby, UK

\subsection{6/gutjnl-2014-307263.458}

Introduction Pain relief following the peri-ampullary injection of Botulinum toxin A may offer a safe alternative to biliary manometry that accurately identifies patients with Type $2 / 3$ Sphincter of Oddi Dysfunction (SOD) who will benefit from endoscopic sphincterotomy.

Methods From 2007 to 2012, patients with probable biliary or pancreatic type 2 or 3 Sphincter of Oddi Dysfunction based on
Rome III revised Milwaukee criteria underwent injection of 100 units Botox divided into four quadrants close to the major papilla.. Response was characterised as complete (CR), partial (at least $50 \%$ improvement) (PR) or no response (NR) using a 10point Likert Scale. Patients that showed either CR or PR were offered an endoscopic sphincterotomy when symptoms returned or worsened.

Results 91patients with median age 39 (22-64), 92.3\% female were studied.

93\% patients with suspected Type 2 biliary SOD $(\mathrm{n}=15)$ had prior cholecystectomy: $73 \%$ had a response to Botox (5CR, 6PR). 7 underwent ES for recurrent symptoms, 2CR and 5PR (100\% responders). The time from Botox to ES ranged from 12 to 42 weeks (median 20 weeks). Of the 7 who underwent ES 4 of the responders had no further intervention, 3 underwent extension of their ES for recurrent symptoms within 5 to 8 months. Of 3 NR to Botox who underwent ES only 1 responded. Of the NR none had any further intervention.

$84 \%$ patients with suspected Type 3 biliary SOD $(n=64)$ had prior cholecystectomy: $53 \%$ had a response to Botox (17CR, 23PR). 26 underwent ES for recurrent symptoms, 8CR and 15PR (88\% responders). Of 13 Botox NR who underwent ES, only 3 responded (23\%). The time range from Botox to ES ranged from 6 to 58 weeks (median of 17.5 weeks). Of the 26 who underwent ES and were CR or PR 18 had no further intervention and 8 had extended ES for recurrent symptoms within 2 to 30 months. Of the 3 responders to ES but not Botox 2 had further ES 1 with CR and 1 NR.

$83 \%$ patients with suspected Type 3 pancreatic SOD $(n=6)$ had prior cholecystectomy: $83 \%$ had a response to Botox (4CR, 1PR). 2 underwent ES within 12 to 19 weeks post Botox, and both responded with one having extension of ES for recurrent symptoms with a PR.

Conclusion Botox injection identifies patients likely to respond to ES avoiding the risks of manometry. Manometry may be helpful in a proportion of Botox non-responder in whom SOD is still suspected.

Disclosure of Interest None Declared.

\section{PTH-013 ROUTINE COAGULATION PROFILE PRIOR TO ERCP - AN UNNECESSARY INVESTIGATION?}

${ }^{1} \mathrm{~L}$ Onos*, ${ }^{2} \mathrm{~A}$ Wyman. ${ }^{1}$ General Surgery, Victoria Infirmary-Glasgow, Glasgow, UK; ${ }^{2}$ Upper GI Surgery, Nothern General Hospital-Sheffield, Sheffield, UK

10.1136/gutjnl-2014-307263.459

Introduction From the first reported endoscopic retrograde cholangiopancreatography (ERCP) in 1968 to the invention of endoscopic sphincterectomy in 1974, ERCP has evolved to an almost entirely therapeutic procedure. It is appreciated that approximately 54,000 patients undergo ERCP each year. ${ }^{1}$ The 2008 British guidelines include routine pre-procedural coagulation screen within 3 days prior to ERCP. ${ }^{2}$ However, the American Society for Gastrointestinal Endoscopy guidelines state that the prothrombin time, International Normalised Ratio (INR), and activated partial thromboplastin time in patients without clinical evidence of bleeding disorder or coagulopathy do not predict or correlate with either intraoperative or postoperative haemorrhage. ${ }^{3}$

Methods A 12-month retrospective study of 440 patients undergoing ERCP in a single UK tertiary institution was undertaken. The correlation between the presence of a biochemical cholestatic picture and the INR was assessed. 
Results The blood results of 440 patients were reviewed using the ICE (Integrated Clinical System). The median age was 67years. Of the 440 patients, 310 had deranged LFTs, 86 had a normal liver profile, and 44 had no LFTs taken prior to ERCP. Of those with deranged LFTs, $2 \%$ had only a raised bilirubin, less than $6 \%$ had either only raised transaminases or both raised transaminases and bilirubin, $12 \%$ had only both raised alkaline phosphatase (ALP) and bilirubin, 15\% had both raised ALP and transaminases, $22 \%$ had only raised ALP, and $43 \%$ had a trio of raised bilirubin, ALP, and transaminases. Only 11 patients had no coagulation profile taken before the procedure. Out of the remaining 429 patients, 15 were on warfarin, 2 had haemophilia, 1 was thrombocytopenic, and 1 had von Willebrand's disease. However, out of 440 patients only 2 had an INR greater than $1.5,1$ of them being on warfarin.

Conclusion These results suggest that routine measurement of coagulation profile is unnecessary. This would reduce time delays, decrease costs and avoid further tests in patients. We suggest that the current pre-ERCP investigation guidelines be reviewed. ${ }^{4}$

\section{REFERENCES}

1 The future of service and training in ERCP in UK-J. R. B. GREEN and The UK ERCP Stakeholders working party- August 2007-British Society of Gastroenterology

2 J Williams, J Green, I Beckingham, R Parks, D Martin, M Lombard-Guidelines on the management of common bile duct stones- Gut 2008;57:1004-1021. doi:10.1136/gut.2007.121657

3 Position statement on routine laboratory testing before endoscopic procedureshttp://www.asge.org

4 John Baillie, PierAlberto Testoni. Are we meeting the standards set for ERCP? Gut. 2007 June; 56(6):744-746. doi: 10.1136/gut.2006.113456

Disclosure of Interest None Declared.

\section{PTH-014 PANCREATIC DUCT DILATATION SHOULD BE INVESTIGATED WITH ENDOSCOPIC ULTRASOUND IF COMPUTERISED TOMOGRAPHY FAILS TO IDENTIFY A LESION}

MTA Roberts*, E Javad, J Iqbal. Gastroenterology, University Hospital South Manchester, Manchester, UK

\subsection{6/gutjnl-2014-307263.460}

Introduction A dilated Pancreatic Duct (PD) may be associated with pancreatic disease, but this is infrequently investigated further if no lesion is found on Computerised Tomography (CT). There is limited data on the role of Endoscopic Ultrasound (EUS) with PD dilatation without a cause on CT, as the literature mostly describes the utility of EUS with apparent pancreatic lesions. We describe our experience of EUS in identification of causative lesions not apparent on CT.

Methods Sixty-one (61) cases were identified and retrospectively reviewed between 2007-2013 by searching the EUS database at a university teaching hospital. All had CT +/- MRCP findings of dilated PD (+/- Common Bile Duct (CBD) dilatation) and no pancreatic lesion (54 patients) or oedema/unable to exclude a lesion (7). Results Mean patient age was 70 (41-90). Indications for CT included abdominal pain 16; abnormal Liver Function Tests (LFT) 14 (3 jaundiced); weight loss 9; other 14 (eg. staging CT for lung ca, CT colonography for diarrhoea etc). Mean PD diameter was $6 \mathrm{~mm}(3-25 \mathrm{~mm})$ and 30 had CBD dilatation. CT showed normal pancreatic parenchyma in $46(76 \%)$; prominent ampulla 5 (8\%); pancreatic cyst 5 (8\%), calcification 4 (6.5\%); pseudocyst $1(1.5 \%)$.
After EUS, 49 (80\%) had PD dilatation confirmed, whilst 31 (51\%) also had CBD dilatation. 38 (62\%) failed to identify a cause and hence agreed with CT. Of the remaining 23 (38\%) there was disparity between CT and EUS. An FNA biopsy was performed in $16(26 \%)$ of cases. Findings included neoplasm 9 (15\%); IPMN 4 (6.5\%); biliary stone disease 3 (5\%); chronic pancreatitis 3 (5\%); pseudocyst $1(1.5 \%)$; choledochal cyst 1 (1.5\%); and pancreas divisum 1 (1.5\%). Neoplastic disease included pancreas cancer 5; suspicious ampullary tumour 2; cholangiocarcinoma 1; and mucinous cystadenoma 1 .

With particular reference to EUS, there was isolated PD dilatation in 27 cases (44\% of total), and abnormalities detected in (59\%) which included cancer or IPMN (15\%). In PD and CBD dilatation $22(36 \%) ; 6$ cases were abnormal of which $4(18 \%)$ had cancer (pancreas and ampulla). Without PD dilatation 12 (20\%), pathology was found in 50\% including cholangiocarcinoma (1), IPMN (1), CBD stones (3), chronic pancreatitis (1). Of 9 cancer patients, dilatation was seen in PD only (4); PD and CBD (4); normal PD or CBD (1).

Conclusion PD dilatation should be investigated further with EUS, even when CT shows no causative lesion. We identified a significant percentage of benign (21\%) and malignant (15\%) pathology with EUS. EUS offers the additional advantage of biopsy when there is diagnostic doubt. Normal LFTs and the absence of the 'double duct sign' are insufficient to exclude neoplastic disease and EUS will help identify these.

Disclosure of Interest None Declared.

\section{PTH-015 POST-ERCP PANCREATITIS IN SECONDARY CARE: CAN WE PREDICT WHO WOULD BENEFIT FROM A PROPHYLACTIC PANCREATIC STENT?}

O Old*, TJ Hardy, D Hewin, H Barr, J Brown. Gloucestershire Hospitals NHS Trust, Gloucester, UK

\subsection{6/gutjnl-2014-307263.461}

Introduction Pancreatitis is a recognised complication of ERCP. Measures taken to reduce the incidence in high-risk patients include placement of prophylactic pancreatic stents and use of NSAIDs, but current practice varies widely. The frequency of post-ERCP pancreatitis (PEP) in unselected groups ranges from $1.3-6.7 \%$ in the largest reported series. Historically, prophylactic stents have not been used at our institution. This retrospective study was designed firstly to measure rates of PEP in our institution (a district general hospital), and secondly to identify those cases which may have benefitted from prophylactic pancreatic stenting.

Methods A retrospective database search identified all patients undergoing ERCP across our Trust over a 5-year period (April 2007 to July 2012). A linked search with a county-wide biochemistry database then identified all those patients who had subsequently developed an elevated serum amylase up to 7 days following ERCP. A consensus grading system was used to define PEP as: clinical pancreatitis (new/worsened abdominal pain), requiring or prolonging hospital admission by $\geq 2$ days, with serum amylase over 3 times upper limit of normal $>24 \mathrm{~h}$ post procedure (300 units/l). Additional risk factors for post-ERCP pancreatitis were identified: previous PEP, Sphincter of Oddi dysfunction, repeated cannulation, injection of contrast into pancreatic duct, Sphincter of Oddi manometry, balloon dilatation, precut sphincterotomy, or pancreatic sphincterotomy.

Results 2699 patients underwent ERCP during the study period. 6 patients were excluded due to incomplete records. 57 patients 